Kragujevac Journal of Mathematics

Volume 41(1) (2017), Pages 33-55.

\title{
SOME TRACE INEQUALITIES FOR OPERATORS IN HILBERT SPACES
}

\author{
SILVESTRU SEVER DRAGOMIR ${ }^{1,2}$
}

\begin{abstract}
Some new trace inequalities for operators in Hilbert spaces are provided. The superadditivity and monotonicity of some associated functionals are investigated and applications for power series of such operators are given. Some trace inequalities for matrices are also derived. Examples for the operator exponential and other similar functions are presented as well.
\end{abstract}

\section{INTRODUCTION}

Let $(H,\langle\cdot, \cdot\rangle)$ be a complex Hilbert space and $\left\{e_{i}\right\}_{i \in I}$ an orthonormal basis of $H$. We say that $A \in \mathcal{B}(H)$ is a Hilbert-Schmidt operator if

$$
\sum_{i \in I}\left\|A e_{i}\right\|^{2}<\infty
$$

It is well know that, if $\left\{e_{i}\right\}_{i \in I}$ and $\left\{f_{j}\right\}_{j \in J}$ are orthonormal bases for $H$ and $A \in \mathcal{B}(H)$ then

$$
\sum_{i \in I}\left\|A e_{i}\right\|^{2}=\sum_{j \in I}\left\|A f_{j}\right\|^{2}=\sum_{j \in I}\left\|A^{*} f_{j}\right\|^{2}
$$

showing that the definition (1.1) is independent of the orthonormal basis and $A$ is a Hilbert-Schmidt operator iff $A^{*}$ is a Hilbert-Schmidt operator.

Key words and phrases. Trace class operators, Hilbert-Schmidt operators, trace, Schwarz inequality, trace inequalities for matrices, power series of operators.

2010 Mathematics Subject Classification. Primary: 47A63. Secondary: 47A99.

Received: May 18, 2016.

Accepted: July 4, 2016. 
Let $\mathcal{B}_{2}(H)$ the set of Hilbert-Schmidt operators in $\mathcal{B}(H)$. For $A \in \mathcal{B}_{2}(H)$ we define

$$
\|A\|_{2}:=\left(\sum_{i \in I}\left\|A e_{i}\right\|^{2}\right)^{\frac{1}{2}}
$$

for $\left\{e_{i}\right\}_{i \in I}$ an orthonormal basis of $H$. This definition does not depend on the choice of the orthonormal basis.

Using the triangle inequality in $l^{2}(I)$, one checks that $\mathcal{B}_{2}(H)$ is a vector space and that $\|\cdot\|_{2}$ is a norm on $\mathcal{B}_{2}(H)$, which is usually called in the literature as the Hilbert-Schmidt norm.

Denote the modulus of an operator $A \in \mathcal{B}(H)$ by $|A|:=\left(A^{*} A\right)^{\frac{1}{2}}$.

Because $\||A| x\|=\|A x\|$ for all $x \in H, A$ is Hilbert-Schmidt iff $|A|$ is HilbertSchmidt and $\|A\|_{2}=\||A|\|_{2}$. From (1.2) we have that if $A \in \mathcal{B}_{2}(H)$, then $A^{*} \in \mathcal{B}_{2}(H)$ and $\|A\|_{2}=\left\|A^{*}\right\|_{2}$.

The following theorem collects some of the most important properties of HilbertSchmidt operators.

Theorem 1.1. We have:

(i) $\left(\mathcal{B}_{2}(H),\|\cdot\|_{2}\right)$ is a Hilbert space with inner product

$$
\langle A, B\rangle_{2}:=\sum_{i \in I}\left\langle A e_{i}, B e_{i}\right\rangle=\sum_{i \in I}\left\langle B^{*} A e_{i}, e_{i}\right\rangle
$$

and the definition does not depend on the choice of the orthonormal basis $\left\{e_{i}\right\}_{i \in I}$;

(ii) We have the inequalities

for any $A \in \mathcal{B}_{2}(H)$ and

$$
\|A\| \leq\|A\|_{2}
$$

$$
\|A T\|_{2},\|T A\|_{2} \leq\|T\|\|A\|_{2}
$$

for any $A \in \mathcal{B}_{2}(H)$ and $T \in \mathcal{B}(H)$;

(iii) $\mathcal{B}_{2}(H)$ is an operator ideal in $\mathcal{B}(H)$, i.e.

$$
\mathcal{B}(H) \mathcal{B}_{2}(H) \mathcal{B}(H) \subseteq \mathcal{B}_{2}(H) ;
$$

(iv) $\mathcal{B}_{\text {fin }}(H)$, the space of operators of finite rank, is a dense subspace of $\mathcal{B}_{2}(H)$;

(v) $\mathcal{B}_{2}(H) \subseteq \mathcal{K}(H)$, where $\mathcal{K}(H)$ denotes the algebra of compact operators on $H$.

If $\left\{e_{i}\right\}_{i \in I}$ an orthonormal basis of $H$, we say that $A \in \mathcal{B}(H)$ is trace class if

$$
\|A\|_{1}:=\sum_{i \in I}\left\langle|A| e_{i}, e_{i}\right\rangle<\infty .
$$

The definition of $\|A\|_{1}$ does not depend on the choice of the orthonormal basis $\left\{e_{i}\right\}_{i \in I}$. We denote by $\mathcal{B}_{1}(H)$ the set of trace class operators in $\mathcal{B}(H)$.

The following proposition holds.

Proposition 1.1. If $A \in \mathcal{B}(H)$, then the following are equivalent:

(i) $A \in \mathcal{B}_{1}(H)$; 
(ii) $|A|^{\frac{1}{2}} \in \mathcal{B}_{2}(H)$;

(iii) $A($ or $|A|)$ is the product of two elements of $\mathcal{B}_{2}(H)$.

The following properties are also well known.

Theorem 1.2. With the above notations:

(i) We have

$$
\|A\|_{1}=\left\|A^{*}\right\|_{1} \text { and }\|A\|_{2} \leq\|A\|_{1}
$$

for any $A \in \mathcal{B}_{1}(H)$;

(ii) $\mathcal{B}_{1}(H)$ is an operator ideal in $\mathcal{B}(H)$, i.e.

$$
\mathcal{B}(H) \mathcal{B}_{1}(H) \mathcal{B}(H) \subseteq \mathcal{B}_{1}(H) ;
$$

(iii) We have

$$
\mathcal{B}_{2}(H) \mathcal{B}_{2}(H)=\mathcal{B}_{1}(H) \text {; }
$$

(iv) We have

$$
\|A\|_{1}=\sup \left\{\left|\langle A, B\rangle_{2}\right| \mid B \in \mathcal{B}_{2}(H),\|B\| \leq 1\right\} ;
$$

(v) $\left(\mathcal{B}_{1}(H),\|\cdot\|_{1}\right)$ is a Banach space;

(vi) We have the following isometric isomorphisms

$$
\mathcal{B}_{1}(H) \cong K(H)^{*} \text { and } \mathcal{B}_{1}(H)^{*} \cong \mathcal{B}(H),
$$

where $K(H)^{*}$ is the dual space of $K(H)$ and $\mathcal{B}_{1}(H)^{*}$ is the dual space of $\mathcal{B}_{1}(H)$.

We define the trace of a trace class operator $A \in \mathcal{B}_{1}(H)$ to be

$$
\operatorname{tr}(A):=\sum_{i \in I}\left\langle A e_{i}, e_{i}\right\rangle
$$

where $\left\{e_{i}\right\}_{i \in I}$ an orthonormal basis of $H$. Note that this coincides with the usual definition of the trace if $H$ is finite-dimensional. We observe that the series (1.3) converges absolutely and it is independent from the choice of basis.

The following result collects some properties of the trace.

Theorem 1.3. We have

(i) If $A \in \mathcal{B}_{1}(H)$ then $A^{*} \in \mathcal{B}_{1}(H)$ and

$$
\operatorname{tr}\left(A^{*}\right)=\overline{\operatorname{tr}(A)}
$$

(ii) If $A \in \mathcal{B}_{1}(H)$ and $T \in \mathcal{B}(H)$, then $A T, T A \in \mathcal{B}_{1}(H)$ and

$$
\operatorname{tr}(A T)=\operatorname{tr}(T A) \text { and }|\operatorname{tr}(A T)| \leq\|A\|_{1}\|T\| ;
$$

(iii) $\operatorname{tr}(\cdot)$ is a bounded linear functional on $\mathcal{B}_{1}(H)$ with $\|\operatorname{tr}\|=1$;

(iv) If $A, B \in \mathcal{B}_{2}(H)$ then $A B, B A \in \mathcal{B}_{1}(H)$ and $\operatorname{tr}(A B)=\operatorname{tr}(B A)$;

(v) $\mathcal{B}_{\text {fin }}(H)$ is a dense subspace of $\mathcal{B}_{1}(H)$. 
Utilizing the trace notation we obviously have that

$$
\langle A, B\rangle_{2}=\operatorname{tr}\left(B^{*} A\right)=\operatorname{tr}\left(A B^{*}\right) \text { and }\|A\|_{2}^{2}=\operatorname{tr}\left(A^{*} A\right)=\operatorname{tr}\left(|A|^{2}\right)
$$

for any $A, B \in \mathcal{B}_{2}(H)$.

Now, for the finite dimensional case, it is well known that the trace functional is submultiplicative, that is, for positive semidefinite matrices $A$ and $B$ in $M_{n}(\mathbb{C})$,

$$
0 \leq \operatorname{tr}(A B) \leq \operatorname{tr}(A) \operatorname{tr}(B) .
$$

Therefore

$$
0 \leq \operatorname{tr}\left(A^{k}\right) \leq[\operatorname{tr}(A)]^{k},
$$

where $k$ is any positive integer.

In 2000, Yang [17] proved a matrix trace inequality

$$
\operatorname{tr}\left[(A B)^{k}\right] \leq(\operatorname{tr} A)^{k}(\operatorname{tr} B)^{k},
$$

where $A$ and $B$ are positive semidefinite matrices over $\mathbb{C}$ of the same order $n$ and $k$ is any positive integer. For related works the reader can refer to $[3,4,11,18]$, which are continuations of the work of Bellman [2].

If $(H,\langle\cdot, \cdot\rangle)$ is a separable infinite-dimensional Hilbert space then the inequality (1.4) is also valid for any positive operators $A, B \in \mathcal{B}_{1}(H)$. This result was obtained by L. Liu in 2007, see [9].

In 2001, Yang et al. [16] improved (1.4) as follows

$$
\operatorname{tr}\left[(A B)^{m}\right] \leq\left[\operatorname{tr}\left(A^{2 m}\right) \operatorname{tr}\left(B^{2 m}\right)\right]^{\frac{1}{2}},
$$

where $A$ and $B$ are positive semidefinite matrices over $\mathbb{C}$ of the same order and $m$ is any positive integer.

In [13] the authors have proved many trace inequalities for sums and products of matrices. For instance, if $A$ and $B$ are positive semidefinite matrices in $M_{n}(\mathbb{C})$ then

$$
\operatorname{tr}\left[(A B)^{k}\right] \leq \min \left\{\|A\|^{k} \operatorname{tr}\left(B^{k}\right),\|B\|^{k} \operatorname{tr}\left(A^{k}\right)\right\}
$$

for any positive integer $k$. Also, if $A, B \in M_{n}(\mathbb{C})$ then for $r \geq 1$ and $p, q>1$ with $1 / p+1 / q=1$ we have the following Young type inequality

$$
\operatorname{tr}\left(\left|A B^{*}\right|^{r}\right) \leq \operatorname{tr}\left[\left(\frac{|A|^{p}}{p}+\frac{|B|^{q}}{q}\right)^{r}\right] .
$$

Ando [1] proved a very strong form of Young's inequality - it was shown that if $A$ and $B$ are in $M_{n}(\mathbb{C})$, then there is a unitary matrix $U$ such that

$$
\left|A B^{*}\right| \leq U\left(\frac{1}{p}|A|^{p}+\frac{1}{q}|B|^{q}\right) U^{*}
$$

where $p, q>1$ with $1 / p+1 / q=1$, which immediately gives the trace inequality

$$
\operatorname{tr}\left(\left|A B^{*}\right|\right) \leq \frac{1}{p} \operatorname{tr}\left(|A|^{p}\right)+\frac{1}{q} \operatorname{tr}\left(|B|^{q}\right) .
$$

This inequality can also be obtained from (1.7) by taking $r=1$. 
Another Hölder type inequality has been proved by Manjegani in [10] and can be stated as follows:

$$
\operatorname{tr}(A B) \leq\left[\operatorname{tr}\left(A^{p}\right)\right]^{\frac{1}{p}}\left[\operatorname{tr}\left(B^{q}\right)\right]^{\frac{1}{q}}
$$

where $p, q>1$ with $1 / p+1 / q=1$ and $A$ and $B$ are positive semidefinite matrices.

For the theory of trace functionals and their applications the reader is referred to [14].

For other trace inequalities see [3, 6-8, 12, 15].

In this paper, motivated by the above results, some new inequalities for HilbertSchmidt operators in $\mathcal{B}(H)$ are provided. The superadditivity and monotonicity of some associated functionals are investigated and applications for power series of such operators are given. Some trace inequalities for matrices are also derived. Examples for the operator exponential and other similar functions are presented as well.

\section{Some Trace Inequalities via Hermitian Forms}

Let $P$ a selfadjoint operator with $P \geq 0$. For $A \in \mathcal{B}_{2}(H)$ and $\left\{e_{i}\right\}_{i \in I}$ an orthonormal basis of $H$ we have

$$
\|A\|_{2, P}^{2}:=\operatorname{tr}\left(A^{*} P A\right)=\sum_{i \in I}\left\langle P A e_{i}, A e_{i}\right\rangle \leq\|P\| \sum_{i \in I}\left\|A e_{i}\right\|^{2}=\|P\|\|A\|_{2}^{2},
$$

which shows that $\langle\cdot, \cdot\rangle_{2, P}$ defined by

$$
\langle A, B\rangle_{2, P}:=\operatorname{tr}\left(B^{*} P A\right)=\sum_{i \in I}\left\langle P A e_{i}, B e_{i}\right\rangle=\sum_{i \in I}\left\langle B^{*} P A e_{i}, e_{i}\right\rangle
$$

is a nonnegative Hermitian form on $\mathcal{B}_{2}(H)$, i.e. $\langle\cdot, \cdot\rangle_{2, P}$ satisfies the properties:

(h) $\langle A, A\rangle_{2, P} \geq 0$ for any $A \in \mathcal{B}_{2}(H)$;

(hh) $\langle\cdot, \cdot\rangle_{2, P}$ is linear in the first variable;

(hhh) $\langle B, A\rangle_{2, P}=\overline{\langle A, B}_{2, P}$ for any $A, B \in \mathcal{B}_{2}(H)$.

Using the properties of the trace we also have the following representations

$$
\|A\|_{2, P}^{2}:=\operatorname{tr}\left(P\left|A^{*}\right|^{2}\right)=\operatorname{tr}\left(\left|A^{*}\right|^{2} P\right)
$$

and

$$
\langle A, B\rangle_{2, P}:=\operatorname{tr}\left(P A B^{*}\right)=\operatorname{tr}\left(A B^{*} P\right)=\operatorname{tr}\left(B^{*} P A\right)
$$

for any $A, B \in \mathcal{B}_{2}(H)$.

We start with the following result.

Theorem 2.1. Let $P$ a selfadjoint operator with $P \geq 0$, i.e. $\langle P x, x\rangle \geq 0$ for any $x \in H$.

(i) For any $A, B \in \mathcal{B}_{2}(H)$ we have

$$
\left|\operatorname{tr}\left(P A B^{*}\right)\right|^{2} \leq \operatorname{tr}\left(P\left|A^{*}\right|^{2}\right) \operatorname{tr}\left(P\left|B^{*}\right|^{2}\right)
$$

and

$$
\left[\operatorname{tr}\left(P\left|A^{*}\right|^{2}\right)+2 \operatorname{Re} \operatorname{tr}\left(P A B^{*}\right)+\operatorname{tr}\left(P\left|B^{*}\right|^{2}\right)\right]^{\frac{1}{2}}
$$




$$
\leq\left[\operatorname{tr}\left(P\left|A^{*}\right|^{2}\right)\right]^{\frac{1}{2}}+\left[\operatorname{tr}\left(P\left|B^{*}\right|^{2}\right)\right]^{\frac{1}{2}}
$$

(ii) For any $A, B, C \in \mathcal{B}_{2}(H)$ we have

$$
\begin{aligned}
& \left|\operatorname{tr}\left(P A B^{*}\right) \operatorname{tr}\left(P\left|C^{*}\right|^{2}\right)-\operatorname{tr}\left(P A C^{*}\right) \operatorname{tr}\left(P C B^{*}\right)\right|^{2} \\
\leq & {\left[\operatorname{tr}\left(P\left|A^{*}\right|^{2}\right) \operatorname{tr}\left(P\left|C^{*}\right|^{2}\right)-\left|\operatorname{tr}\left(P A C^{*}\right)\right|^{2}\right] } \\
& \times\left[\operatorname{tr}\left(P\left|B^{*}\right|^{2}\right) \operatorname{tr}\left(P\left|C^{*}\right|^{2}\right)-\left|\operatorname{tr}\left(P B C^{*}\right)\right|^{2}\right],
\end{aligned}
$$

$$
\begin{aligned}
& \left|\operatorname{tr}\left(P A B^{*}\right)\right| \operatorname{tr}\left(P\left|C^{*}\right|^{2}\right) \\
\leq & \left|\operatorname{tr}\left(P A B^{*}\right) \operatorname{tr}\left(P\left|C^{*}\right|^{2}\right)-\operatorname{tr}\left(P A C^{*}\right) \operatorname{tr}\left(P C B^{*}\right)\right|+\left|\operatorname{tr}\left(P A C^{*}\right) \operatorname{tr}\left(P C B^{*}\right)\right| \\
\leq & {\left[\operatorname{tr}\left(P\left|A^{*}\right|^{2}\right)\right]^{\frac{1}{2}}\left[\operatorname{tr}\left(P\left|B^{*}\right|^{2}\right)\right]^{\frac{1}{2}} \operatorname{tr}\left(P\left|C^{*}\right|^{2}\right) }
\end{aligned}
$$

and

$$
\begin{aligned}
& \left|\operatorname{tr}\left(P A C^{*}\right) \operatorname{tr}\left(P C B^{*}\right)\right| \\
\leq & \frac{1}{2}\left[\left[\operatorname{tr}\left(P\left|A^{*}\right|^{2}\right)\right]^{\frac{1}{2}}\left[\operatorname{tr}\left(P\left|B^{*}\right|^{2}\right)\right]^{\frac{1}{2}}+\left|\operatorname{tr}\left(P A B^{*}\right)\right|\right] \operatorname{tr}\left(P\left|C^{*}\right|^{2}\right) .
\end{aligned}
$$

Proof.

(i) Making use of the Schwarz inequality for the nonnegative hermitian form $\langle\cdot, \cdot\rangle_{2, P}$ we have

$$
\left|\langle A, B\rangle_{2, P}\right|^{2} \leq\langle A, A\rangle_{2, P}\langle B, B\rangle_{2, P}
$$

for any $A, B \in \mathcal{B}_{2}(H)$ and the inequality (2.1) is proved.

We observe that $\|\cdot\|_{2, P}$ is a seminorm on $\mathcal{B}_{2}(H)$ and by the triangle inequality we have

$$
\|A+B\|_{2, P} \leq\|A\|_{2, P}+\|B\|_{2, P}
$$

for any $A, B \in \mathcal{B}_{2}(H)$ and the inequality (2.2) is proved.

(ii) Let $C \in \mathcal{B}_{2}(H), C \neq 0$. Define the mapping $[\cdot, \cdot]_{2, P, C}: \mathcal{B}_{2}(H) \times \mathcal{B}_{2}(H) \rightarrow \mathbb{C}$ by

$$
[A, B]_{2, P, C}:=\langle A, B\rangle_{2, P}\|C\|_{2, P}^{2}-\langle A, C\rangle_{2, P}\langle C, B\rangle_{2, P} .
$$

Observe that $[\cdot, \cdot]_{2, P, C}$ is a nonnegative Hermitian form on $\mathcal{B}_{2}(H)$ and by Schwarz inequality we have

$$
\begin{aligned}
& \left|\langle A, B\rangle_{2, P}\|C\|_{2, P}^{2}-\langle A, C\rangle_{2, P}\langle C, B\rangle_{2, P}\right|^{2} \\
\leq & {\left[\|A\|_{2, P}^{2}\|C\|_{2, P}^{2}-\left|\langle A, C\rangle_{2, P}\right|^{2}\right]\left[\|B\|_{2, P}^{2}\|C\|_{2, P}^{2}-\left|\langle B, C\rangle_{2, P}\right|^{2}\right] }
\end{aligned}
$$

for any $A, B \in \mathcal{B}_{2}(H)$, which proves (2.3).

The case $C=0$ is obvious.

Utilizing the elementary inequality for real numbers $m, n, p, q$

$$
\left(m^{2}-n^{2}\right)\left(p^{2}-q^{2}\right) \leq(m p-n q)^{2},
$$


we can easily see that

$$
\begin{aligned}
& {\left[\|A\|_{2, P}^{2}\|C\|_{2, P}^{2}-\left|\langle A, C\rangle_{2, P}\right|^{2}\right]\left[\|B\|_{2, P}^{2}\|C\|_{2, P}^{2}-\left|\langle B, C\rangle_{2, P}\right|^{2}\right] } \\
\leq & \left(\|A\|_{2, P}\|B\|_{2, P}\|C\|_{2, P}^{2}-\left|\langle A, C\rangle_{2, P}\right|\left|\langle B, C\rangle_{2, P}\right|\right)^{2}
\end{aligned}
$$

for any $A, B, C \in \mathcal{B}_{2}(H)$.

Since, by Schwarz's inequality we have

$$
\|A\|_{2, P}\|C\|_{2, P} \geq\left|\langle A, C\rangle_{2, P}\right|
$$

and

$$
\|B\|_{2, P}\|C\|_{2, P} \geq\left|\langle B, C\rangle_{2, P}\right|
$$

then by multiplying these inequalities we have

$$
\|A\|_{2, P}\|B\|_{2, P}\|C\|_{2, P}^{2} \geq\left|\langle A, C\rangle_{2, P}\right|\left|\langle B, C\rangle_{2, P}\right|,
$$

for any $A, B, C \in \mathcal{B}_{2}(H)$.

Utilizing the inequalities (2.6) and (2.7) and taking the square root we get

$$
\begin{aligned}
& \left|\langle A, B\rangle_{2, P}\|C\|_{2, P}^{2}-\langle A, C\rangle_{2, P}\langle C, B\rangle_{2, P}\right| \\
\leq & \|A\|_{2, P}\|B\|_{2, P}\|C\|_{2, P}^{2}-\left|\langle A, C\rangle_{2, P}\right|\left|\langle B, C\rangle_{2, P}\right|
\end{aligned}
$$

for any $A, B, C \in \mathcal{B}_{2}(H)$, which proves the second inequality in (2.4).

The first inequality is obvious by the modulus properties.

By the triangle inequality for modulus we also have

$$
\begin{aligned}
& \left|\langle A, C\rangle_{2, P}\langle C, B\rangle_{2, P}\right|-\left|\langle A, B\rangle_{2, P}\right|\|C\|_{2, P}^{2} \\
\leq & \left|\langle A, B\rangle_{2, P}\|C\|_{2, P}^{2}-\langle A, C\rangle_{2, P}\langle C, B\rangle_{2, P}\right|
\end{aligned}
$$

for any $A, B, C \in \mathcal{B}_{2}(H)$.

On making use of (2.8) and (2.9) we have

$$
\begin{aligned}
& \left|\langle A, C\rangle_{2, P}\langle C, B\rangle_{2, P}\right|-\left|\langle A, B\rangle_{2, P}\right|\|C\|_{2, P}^{2} \\
\leq & \|A\|_{2, P}\|B\|_{2, P}\|C\|_{2, P}^{2}-\left|\langle A, C\rangle_{2, P}\right|\left|\langle B, C\rangle_{2, P}\right|,
\end{aligned}
$$

which is equivalent to the desired inequality (2.5).

Remark 2.1. By the triangle inequality for the hermitian form $[\cdot, \cdot]_{2, P, C}: \mathcal{B}_{2}(H) \times$ $\mathcal{B}_{2}(H) \rightarrow \mathbb{C}$,

$$
[A, B]_{2, P, C}:=\langle A, B\rangle_{2, P}\|C\|_{2, P}^{2}-\langle A, C\rangle_{2, P}\langle C, B\rangle_{2, P}
$$


we have

$$
\begin{aligned}
& \left(\|A+B\|_{2, P}^{2}\|C\|_{2, P}^{2}-\left|\langle A+B, C\rangle_{2, P}\right|^{2}\right)^{\frac{1}{2}} \\
\leq & \left(\|A\|_{2, P}^{2}\|C\|_{2, P}^{2}-\left|\langle A, C\rangle_{2, P}\right|^{2}\right)^{\frac{1}{2}}+\left(\|B\|_{2, P}^{2}\|C\|_{2, P}^{2}-\left|\langle B, C\rangle_{2, P}\right|^{2}\right)^{\frac{1}{2}},
\end{aligned}
$$

which can be written as

$$
\begin{aligned}
& \left(\operatorname{tr}\left[P\left|(A+B)^{*}\right|^{2}\right] \operatorname{tr}\left(P\left|C^{*}\right|^{2}\right)-\left|\operatorname{tr}\left[P(A+B) C^{*}\right]\right|^{2}\right)^{\frac{1}{2}} \\
\leq & \left(\operatorname{tr}\left(P\left|A^{*}\right|^{2}\right) \operatorname{tr}\left(P\left|C^{*}\right|^{2}\right)-\left|\operatorname{tr}\left(P A C^{*}\right)\right|^{2}\right)^{\frac{1}{2}} \\
& +\left(\operatorname{tr}\left(P\left|B^{*}\right|^{2}\right) \operatorname{tr}\left(P\left|C^{*}\right|^{2}\right)-\left|\operatorname{tr}\left(P B C^{*}\right)\right|^{2}\right)^{\frac{1}{2}}
\end{aligned}
$$

for any $A, B, C \in \mathcal{B}_{2}(H)$.

Remark 2.2. If we take $B=\lambda C$ in (2.10), then we get

$$
\begin{aligned}
0 & \leq \operatorname{tr}\left[P\left|(A+\lambda C)^{*}\right|^{2}\right] \operatorname{tr}\left(P\left|C^{*}\right|^{2}\right)-\left|\operatorname{tr}\left[P(A+\lambda C) C^{*}\right]\right|^{2} \\
& \leq \operatorname{tr}\left(P\left|A^{*}\right|^{2}\right) \operatorname{tr}\left(P\left|C^{*}\right|^{2}\right)-\left|\operatorname{tr}\left(C^{*} P A\right)\right|^{2},
\end{aligned}
$$

for any $\lambda \in \mathbb{C}$ and $A, C \in \mathcal{B}_{2}(H)$.

Therefore, we have the bound

$$
\begin{aligned}
& \sup _{\lambda \in \mathbb{C}}\left\{\operatorname{tr}\left[P\left|(A+\lambda C)^{*}\right|^{2}\right] \operatorname{tr}\left(P\left|C^{*}\right|^{2}\right)-\left|\operatorname{tr}\left[P(A+\lambda C) C^{*}\right]\right|^{2}\right\} \\
= & \operatorname{tr}\left(P\left|A^{*}\right|^{2}\right) \operatorname{tr}\left(P\left|C^{*}\right|^{2}\right)-\left|\operatorname{tr}\left(P A C^{*}\right)\right|^{2} .
\end{aligned}
$$

We also have the inequalities

$$
\begin{aligned}
0 & \leq \operatorname{tr}\left[P\left|(A \pm C)^{*}\right|^{2}\right] \operatorname{tr}\left(P\left|C^{*}\right|^{2}\right)-\left|\operatorname{tr}\left[P(A \pm C) C^{*}\right]\right|^{2} \\
& \leq \operatorname{tr}\left(P\left|A^{*}\right|^{2}\right) \operatorname{tr}\left(P\left|C^{*}\right|^{2}\right)-\left|\operatorname{tr}\left(P A C^{*}\right)\right|^{2},
\end{aligned}
$$

for any $A, C \in \mathcal{B}_{2}(H)$.

Remark 2.3. We observe that, by replacing $A^{*}$ with $A, B^{*}$ with $B$ etc. above, we can get the dual inequalities, like, for instance

$$
\begin{aligned}
& \left|\operatorname{tr}\left(P A^{*} C\right) \operatorname{tr}\left(P C^{*} B\right)\right| \\
\leq & \frac{1}{2}\left[\left[\operatorname{tr}\left(P|A|^{2}\right)\right]^{\frac{1}{2}}\left[\operatorname{tr}\left(P|B|^{2}\right)\right]^{\frac{1}{2}}+\left|\operatorname{tr}\left(P A^{*} B\right)\right|\right] \operatorname{tr}\left(P|C|^{2}\right),
\end{aligned}
$$

that holds for any $A, B, C \in \mathcal{B}_{2}(H)$.

Since

$$
\begin{aligned}
& \left|\operatorname{tr}\left(P A^{*} C\right)\right|=\left|\overline{\operatorname{tr}\left(P A^{*} C\right)}\right|=\left|\operatorname{tr}\left[\left(P A^{*} C\right)^{*}\right]\right|=\left|\operatorname{tr}\left(C^{*} A P\right)\right|=\left|\operatorname{tr}\left(P C^{*} A\right)\right|, \\
& \left|\operatorname{tr}\left(P C^{*} B\right)\right|=\left|\operatorname{tr}\left(P B^{*} C\right)\right|
\end{aligned}
$$


and

$$
\left|\operatorname{tr}\left(P A^{*} B\right)\right|=\left|\operatorname{tr}\left(P B^{*} A\right)\right|
$$

then the inequality $(2.14)$ can be also written as

$$
\begin{aligned}
& \left|\operatorname{tr}\left(P C^{*} A\right) \operatorname{tr}\left(P B^{*} C\right)\right| \\
\leq & \frac{1}{2}\left[\left[\operatorname{tr}\left(P|A|^{2}\right)\right]^{\frac{1}{2}}\left[\operatorname{tr}\left(P|B|^{2}\right)\right]^{\frac{1}{2}}+\left|\operatorname{tr}\left(P B^{*} A\right)\right|\right] \operatorname{tr}\left(P|C|^{2}\right),
\end{aligned}
$$

that holds for any $A, B, C \in \mathcal{B}_{2}(H)$.

If we take in (2.15) $B=A^{*}$ then we get the following inequality

$$
\begin{aligned}
& \left|\operatorname{tr}\left(P C^{*} A\right) \operatorname{tr}(P A C)\right| \\
\leq & \frac{1}{2}\left[\left[\operatorname{tr}\left(P|A|^{2}\right)\right]^{\frac{1}{2}}\left[\operatorname{tr}\left(P\left|A^{*}\right|^{2}\right)\right]^{\frac{1}{2}}+\left|\operatorname{tr}\left(P A^{2}\right)\right|\right] \operatorname{tr}\left(P|C|^{2}\right),
\end{aligned}
$$

for any $A, B, C \in \mathcal{B}_{2}(H)$.

If $A$ is a normal operator, i.e. $|A|^{2}=\left|A^{*}\right|^{2}$ then we have from (2.16) that

$$
\left|\operatorname{tr}\left(P C^{*} A\right) \operatorname{tr}(P A C)\right| \leq \frac{1}{2}\left[\operatorname{tr}\left(P|A|^{2}\right)+\left|\operatorname{tr}\left(P A^{2}\right)\right|\right] \operatorname{tr}\left(P|C|^{2}\right),
$$

In particular, if $C$ is selfadjoint and $C \in \mathcal{B}_{2}(H)$, then

$$
|\operatorname{tr}(P A C)|^{2} \leq \frac{1}{2}\left[\operatorname{tr}\left(P|A|^{2}\right)+\left|\operatorname{tr}\left(P A^{2}\right)\right|\right] \operatorname{tr}\left(P C^{2}\right)
$$

for any $A \in \mathcal{B}_{2}(H)$ a normal operator.

We notice that (2.17) is a trace operator version of de Bruijn inequality obtained in 1960 in [5], which gives the following refinement of the Cauchy-Bunyakovsky-Schwarz inequality

$$
\left|\sum_{i=1}^{n} a_{i} z_{i}\right|^{2} \leq \frac{1}{2} \sum_{i=1}^{n} a_{i}^{2}\left[\sum_{i=1}^{n}\left|z_{i}\right|^{2}+\left|\sum_{i=1}^{n} z_{i}^{2}\right|\right],
$$

provided that $a_{i}$ are real numbers while $z_{i}$ are complex for each $i \in\{1, \ldots, n\}$.

We notice that, if $P \in \mathcal{B}_{1}(H), P \geq 0$ and $A, B \in \mathcal{B}(H)$, then

$$
\langle A, B\rangle_{2, P}:=\operatorname{tr}\left(P A B^{*}\right)=\operatorname{tr}\left(A B^{*} P\right)=\operatorname{tr}\left(B^{*} P A\right)
$$

is a nonnegative Hermitian form on $\mathcal{B}(H)$ and all the inequalities above will hold for $A, B, C \in \mathcal{B}(H)$. The details are left to the reader.

\section{Some Functional Properties}

We consider now the convex cone $\mathcal{B}_{+}(H)$ of nonnegative operators on the complex Hilbert space $H$ and, for $A, B \in \mathcal{B}_{2}(H)$ define the functional $\sigma_{A, B}: \mathcal{B}_{+}(H) \rightarrow[0, \infty)$ by

$$
\sigma_{A, B}(P):=\left[\operatorname{tr}\left(P|A|^{2}\right)\right]^{\frac{1}{2}}\left[\operatorname{tr}\left(P|B|^{2}\right)\right]^{\frac{1}{2}}-\left|\operatorname{tr}\left(P A^{*} B\right)\right|(\geq 0) .
$$

The following theorem collects some fundamental properties of this functional. 
Theorem 3.1. Let $A, B \in \mathcal{B}_{2}(H)$.

(i) For any $P, Q \in \mathcal{B}_{+}(H)$ we have

$$
\sigma_{A, B}(P+Q) \geq \sigma_{A, B}(P)+\sigma_{A, B}(Q)(\geq 0),
$$

namely, $\sigma_{A, B}$ is a superadditive functional on $\mathcal{B}_{+}(H)$;

(ii) For any $P, Q \in \mathcal{B}_{+}(H)$ with $P \geq Q$ we have

$$
\sigma_{A, B}(P) \geq \sigma_{A, B}(Q)(\geq 0)
$$

namely, $\sigma_{A, B}$ is a monotonic nondecreasing functional on $\mathcal{B}_{+}(H)$;

(iii) If $P, Q \in \mathcal{B}_{+}(H)$ and there exist the constants $M>m>0$ such that $M Q \geq$ $P \geq m Q$ then

$$
M \sigma_{A, B}(Q) \geq \sigma_{A, B}(P) \geq m \sigma_{A, B}(Q)(\geq 0)
$$

Proof.

(i) Let $P, Q \in \mathcal{B}_{+}(H)$. On utilizing the elementary inequality

$$
\left(a^{2}+b^{2}\right)^{\frac{1}{2}}\left(c^{2}+d^{2}\right)^{\frac{1}{2}} \geq a c+b d, \quad a, b, c, d \geq 0
$$

and the triangle inequality for the modulus, we have

$$
\begin{aligned}
& \sigma_{A, B}(P+Q) \\
= & {\left[\operatorname{tr}\left((P+Q)|A|^{2}\right)\right]^{\frac{1}{2}}\left[\operatorname{tr}\left((P+Q)|B|^{2}\right)\right]^{\frac{1}{2}}-\left|\operatorname{tr}\left((P+Q) A^{*} B\right)\right| } \\
= & {\left[\operatorname{tr}\left(P|A|^{2}+Q|A|^{2}\right)\right]^{\frac{1}{2}}\left[\operatorname{tr}\left(P|B|^{2}+Q|B|^{2}\right)\right]^{\frac{1}{2}}-\left|\operatorname{tr}\left(P A^{*} B+Q A^{*} B\right)\right| } \\
= & {\left[\operatorname{tr}\left(P|A|^{2}\right)+\operatorname{tr}\left(Q|A|^{2}\right)\right]^{\frac{1}{2}}\left[\operatorname{tr}\left(P|B|^{2}\right)+\operatorname{tr}\left(Q|B|^{2}\right)\right]^{\frac{1}{2}}-\left|\operatorname{tr}\left(P A^{*} B\right)+\operatorname{tr}\left(Q A^{*} B\right)\right| } \\
\geq & {\left[\operatorname{tr}\left(P|A|^{2}\right)\right]^{\frac{1}{2}}\left[\operatorname{tr}\left(P|B|^{2}\right)\right]^{\frac{1}{2}}+\left[\operatorname{tr}\left(Q|A|^{2}\right)\right]^{\frac{1}{2}}\left[\operatorname{tr}\left(Q|B|^{2}\right)\right]^{\frac{1}{2}} } \\
& -\left|\operatorname{tr}\left(P A^{*} B\right)\right|-\left|\operatorname{tr}\left(Q A^{*} B\right)\right| \\
= & \sigma_{A, B}(P)+\sigma_{A, B}(Q)
\end{aligned}
$$

and the inequality (3.2) is proved.

(ii) Let $P, Q \in \mathcal{B}_{+}(H)$ with $P \geq Q$. Utilizing the superadditivity property we have

$$
\sigma_{A, B}(P)=\sigma_{A, B}((P-Q)+Q) \geq \sigma_{A, B}(P-Q)+\sigma_{A, B}(Q) \geq \sigma_{A, B}(Q)
$$

and the inequality (3.3) is obtained.

(iii) From the monotonicity property we have

$$
\sigma_{A, B}(P) \geq \sigma_{A, B}(m Q)=m \sigma_{A, B}(Q)
$$

and a similar inequality for $M$, which prove the desired result (3.4).

Corollary 3.1. Let $A, B \in \mathcal{B}_{2}(H)$ and $P \in \mathcal{B}(H)$ such that there exist the constants $M>m>0$ with $M 1_{H} \geq P \geq m 1_{H}$. Then we have

$$
M\left(\left[\operatorname{tr}\left(|A|^{2}\right)\right]^{\frac{1}{2}}\left[\operatorname{tr}\left(|B|^{2}\right)\right]^{\frac{1}{2}}-\left|\operatorname{tr}\left(A^{*} B\right)\right|\right)
$$




$$
\begin{aligned}
& \geq\left[\operatorname{tr}\left(P|A|^{2}\right)\right]^{\frac{1}{2}}\left[\operatorname{tr}\left(P|B|^{2}\right)\right]^{\frac{1}{2}}-\left|\operatorname{tr}\left(P A^{*} B\right)\right| \\
& \geq m\left(\left[\operatorname{tr}\left(|A|^{2}\right)\right]^{\frac{1}{2}}\left[\operatorname{tr}\left(|B|^{2}\right)\right]^{\frac{1}{2}}-\left|\operatorname{tr}\left(A^{*} B\right)\right|\right) .
\end{aligned}
$$

Let $P=|V|^{2}$ with $V \in \mathcal{B}(H)$. If $A, B \in \mathcal{B}_{2}(H)$ then

$$
\begin{aligned}
\sigma_{A, B}\left(|V|^{2}\right) & =\left[\operatorname{tr}\left(|V|^{2}|A|^{2}\right)\right]^{\frac{1}{2}}\left[\operatorname{tr}\left(|V|^{2}|B|^{2}\right)\right]^{\frac{1}{2}}-\left|\operatorname{tr}\left(|V|^{2} A^{*} B\right)\right| \\
& =\left[\operatorname{tr}\left(V^{*} V A^{*} A\right)\right]^{\frac{1}{2}}\left[\operatorname{tr}\left(V^{*} V B^{*} B\right)\right]^{\frac{1}{2}}-\left|\operatorname{tr}\left(V^{*} V A^{*} B\right)\right| \\
& =\left[\operatorname{tr}\left(V A^{*} A V^{*}\right)\right]^{\frac{1}{2}}\left[\operatorname{tr}\left(V B^{*} B V^{*}\right)\right]^{\frac{1}{2}}-\left|\operatorname{tr}\left(V A^{*} B V^{*}\right)\right| \\
& =\left[\operatorname{tr}\left(\left(A V^{*}\right)^{*} A V^{*}\right)\right]^{\frac{1}{2}}\left[\operatorname{tr}\left(\left(B V^{*}\right)^{*} B V^{*}\right)\right]^{\frac{1}{2}}-\left|\operatorname{tr}\left(\left(A V^{*}\right)^{*} B V^{*}\right)\right| \\
& =\left[\operatorname{tr}\left(\left|A V^{*}\right|^{2}\right)\right]^{\frac{1}{2}}\left[\operatorname{tr}\left(\left|B V^{*}\right|^{2}\right)\right]^{\frac{1}{2}}-\left|\operatorname{tr}\left(\left(A V^{*}\right)^{*} B V^{*}\right)\right| .
\end{aligned}
$$

On utilizing the property (3.2) for $P=|V|^{2}, Q=|U|^{2}$ with $V, U \in \mathcal{B}(H)$, then we have for any $A, B \in \mathcal{B}_{2}(H)$ the following trace inequality

$$
\begin{aligned}
& {\left[\operatorname{tr}\left(\left|A V^{*}\right|^{2}+\left|A U^{*}\right|^{2}\right)\right]^{\frac{1}{2}}\left[\operatorname{tr}\left(\left|B V^{*}\right|^{2}+\left|B U^{*}\right|^{2}\right)\right]^{\frac{1}{2}} } \\
& -\left|\operatorname{tr}\left(\left(A V^{*}\right)^{*} B V^{*}+\left(A U^{*}\right)^{*} B U^{*}\right)\right| \\
\geq & {\left[\operatorname{tr}\left(\left|A V^{*}\right|^{2}\right)\right]^{\frac{1}{2}}\left[\operatorname{tr}\left(\left|B V^{*}\right|^{2}\right)\right]^{\frac{1}{2}}-\left|\operatorname{tr}\left(\left(A V^{*}\right)^{*} B V^{*}\right)\right| } \\
& +\left[\operatorname{tr}\left(\left|A U^{*}\right|^{2}\right)\right]^{\frac{1}{2}}\left[\operatorname{tr}\left(\left|B U^{*}\right|^{2}\right)\right]^{\frac{1}{2}}-\left|\operatorname{tr}\left(\left(A U^{*}\right)^{*} B U^{*}\right)\right|(\geq 0) .
\end{aligned}
$$

Also, if $|V|^{2} \geq|U|^{2}$ with $V, U \in \mathcal{B}(H)$, then we have for any $A, B \in \mathcal{B}_{2}(H)$ that

$$
\begin{aligned}
& {\left[\operatorname{tr}\left(\left|A V^{*}\right|^{2}\right)\right]^{\frac{1}{2}}\left[\operatorname{tr}\left(\left|B V^{*}\right|^{2}\right)\right]^{\frac{1}{2}}-\left|\operatorname{tr}\left(\left(A V^{*}\right)^{*} B V^{*}\right)\right| } \\
\geq & {\left[\operatorname{tr}\left(\left|A U^{*}\right|^{2}\right)\right]^{\frac{1}{2}}\left[\operatorname{tr}\left(\left|B U^{*}\right|^{2}\right)\right]^{\frac{1}{2}}-\left|\operatorname{tr}\left(\left(A U^{*}\right)^{*} B U^{*}\right)\right|(\geq 0) . }
\end{aligned}
$$

If $U \in \mathcal{B}(H)$ is invertible, then

$$
\frac{1}{\left\|U^{-1}\right\|}\|x\| \leq\|U x\| \leq\|U\|\|x\|, \quad \text { for any } x \in H
$$

which implies that

$$
\frac{1}{\left\|U^{-1}\right\|^{2}} 1_{H} \leq|U|^{2} \leq\|U\|^{2} 1_{H}
$$

By making use of (3.5) we have the following trace inequality

$$
\begin{aligned}
& \|U\|^{2}\left(\left[\operatorname{tr}\left(|A|^{2}\right)\right]^{\frac{1}{2}}\left[\operatorname{tr}\left(|B|^{2}\right)\right]^{\frac{1}{2}}-\left|\operatorname{tr}\left(A^{*} B\right)\right|\right) \\
\geq & {\left[\operatorname{tr}\left(\left|A U^{*}\right|^{2}\right)\right]^{\frac{1}{2}}\left[\operatorname{tr}\left(\left|B U^{*}\right|^{2}\right)\right]^{\frac{1}{2}}-\left|\operatorname{tr}\left(\left(A U^{*}\right)^{*} B U^{*}\right)\right| } \\
\geq & \frac{1}{\left\|U^{-1}\right\|^{2}}\left(\left[\operatorname{tr}\left(|A|^{2}\right)\right]^{\frac{1}{2}}\left[\operatorname{tr}\left(|B|^{2}\right)\right]^{\frac{1}{2}}-\left|\operatorname{tr}\left(A^{*} B\right)\right|\right)
\end{aligned}
$$

for any $A, B \in \mathcal{B}_{2}(H)$. 
Similar results may be stated for $P \in \mathcal{B}_{1}(H), P \geq 0$ and $A, B \in \mathcal{B}(H)$. The details are omitted.

\section{Inequalities for Sequences of Operators}

For $n \geq 2$, define the Cartesian products $\mathcal{B}^{(n)}(H):=\mathcal{B}(H) \times \cdots \times \mathcal{B}(H)$, $\mathcal{B}_{2}^{(n)}(H):=\mathcal{B}_{2}(H) \times \cdots \times \mathcal{B}_{2}(H)$ and $\mathcal{B}_{+}^{(n)}(H):=\mathcal{B}_{+}(H) \times \cdots \times \mathcal{B}_{+}(H)$, where $\mathcal{B}_{+}(H)$ denotes the convex cone of nonnegative selfadjoint operators on $H$, i.e. $P \in \mathcal{B}_{+}(H)$ if $\langle P x, x\rangle \geq 0$ for any $x \in H$.

Proposition 4.1. Let $\mathbf{P}=\left(P_{1}, \ldots, P_{n}\right) \in \mathcal{B}_{+}^{(n)}(H)$ and $\mathbf{A}=\left(A_{1}, \ldots, A_{n}\right)$, $\mathbf{B}=$ $\left(B_{1}, \ldots, B_{n}\right) \in \mathcal{B}_{2}^{(n)}(H)$ and $\mathbf{z}=\left(z_{1}, \ldots, z_{n}\right) \in \mathbb{C}^{n}$ with $n \geq 2$. Then

$$
\left|\operatorname{tr}\left(\sum_{k=1}^{n} z_{k} P_{k} A_{k}^{*} B_{k}\right)\right|^{2} \leq \operatorname{tr}\left(\sum_{k=1}^{n}\left|z_{k}\right| P_{k}\left|A_{k}\right|^{2}\right) \operatorname{tr}\left(\sum_{k=1}^{n}\left|z_{k}\right| P_{k}\left|B_{k}\right|^{2}\right) .
$$

Proof. Using the properties of modulus and the inequality (2.1) we have

$$
\begin{aligned}
\left|\operatorname{tr}\left(\sum_{k=1}^{n} z_{k} P_{k} A_{k}^{*} B_{k}\right)\right| & =\left|\sum_{k=1}^{n} z_{k} \operatorname{tr}\left(P_{k} A_{k}^{*} B_{k}\right)\right| \\
& \leq \sum_{k=1}^{n}\left|z_{k}\right|\left|\operatorname{tr}\left(P_{k} A_{k}^{*} B_{k}\right)\right| \\
& \leq \sum_{k=1}^{n}\left|z_{k}\right|\left[\operatorname{tr}\left(P_{k}\left|A_{k}\right|^{2}\right)\right]^{\frac{1}{2}}\left[\operatorname{tr}\left(P_{k}\left|B_{k}\right|^{2}\right)\right]^{\frac{1}{2}} .
\end{aligned}
$$

Utilizing the weighted discrete Cauchy-Bunyakovsky-Schwarz inequality we also have

$$
\begin{aligned}
& \sum_{k=1}^{n}\left|z_{k}\right|\left[\operatorname{tr}\left(P_{k}\left|A_{k}\right|^{2}\right)\right]^{\frac{1}{2}}\left[\operatorname{tr}\left(P_{k}\left|B_{k}\right|^{2}\right)\right]^{\frac{1}{2}} \\
\leq & \left(\sum_{k=1}^{n}\left|z_{k}\right|\left(\left[\operatorname{tr}\left(P_{k}\left|A_{k}\right|^{2}\right)\right]^{\frac{1}{2}}\right)^{2}\right)^{\frac{1}{2}}\left(\sum_{k=1}^{n}\left|z_{k}\right|\left(\left[\operatorname{tr}\left(P_{k}\left|B_{k}\right|^{2}\right)\right]^{\frac{1}{2}}\right)^{2}\right)^{\frac{1}{2}} \\
= & \left(\sum_{k=1}^{n}\left|z_{k}\right| \operatorname{tr}\left(P_{k}\left|A_{k}\right|^{2}\right)\right)^{\frac{1}{2}}\left(\sum_{k=1}^{n}\left|z_{k}\right| \operatorname{tr}\left(P_{k}\left|B_{k}\right|^{2}\right)\right)^{\frac{1}{2}} \\
= & \left(\operatorname{tr}\left(\sum_{k=1}^{n}\left|z_{k}\right| P_{k}\left|A_{k}\right|^{2}\right)\right)^{\frac{1}{2}}\left(\operatorname{tr}\left(\sum_{k=1}^{n}\left|z_{k}\right| P_{k}\left|B_{k}\right|^{2}\right)\right)^{\frac{1}{2}},
\end{aligned}
$$

which is equivalent to the desired result (4.1). 
We consider the functional for $n$-tuples of nonnegative operators as follows:

$$
\begin{aligned}
\sigma_{\mathbf{A}, \mathbf{B}}(\mathbf{P}):= & {\left[\operatorname{tr}\left(\sum_{k=1}^{n} P_{k}\left|A_{k}\right|^{2}\right)\right]^{\frac{1}{2}}\left[\operatorname{tr}\left(\sum_{k=1}^{n} P_{k}\left|B_{k}\right|^{2}\right)\right]^{\frac{1}{2}} } \\
& -\left|\operatorname{tr}\left(\sum_{k=1}^{n} P_{k} A_{k}^{*} B_{k}\right)\right| .
\end{aligned}
$$

Utilizing a similar argument to the one in Theorem 3.1 we can state the following.

Proposition 4.2. Let $\mathbf{A}=\left(A_{1}, \ldots, A_{n}\right), \mathbf{B}=\left(B_{1}, \ldots, B_{n}\right) \in \mathcal{B}_{2}^{(n)}(H)$.

(i) For any $\mathbf{P}, \mathbf{Q} \in \mathcal{B}_{+}^{(n)}(H)$ we have

$$
\sigma_{\mathbf{A}, \mathbf{B}}(\mathbf{P}+\mathbf{Q}) \geq \sigma_{\mathbf{A}, \mathbf{B}}(\mathbf{P})+\sigma_{\mathbf{A}, \mathbf{B}}(\mathbf{Q})(\geq 0),
$$

namely, $\sigma_{\mathbf{A}, \mathbf{B}}$ is a superadditive functional on $\mathcal{B}_{+}^{(n)}(H)$;

(ii) For any $\mathbf{P}, \mathbf{Q} \in \mathcal{B}_{+}^{(n)}(H)$ with $\mathbf{P} \geq \mathbf{Q}$, namely $P_{k} \geq Q_{k}$ for all $k \in\{1, \ldots, n\}$ we have

$$
\sigma_{\mathbf{A}, \mathbf{B}}(\mathbf{P}) \geq \sigma_{\mathbf{A}, \mathbf{B}}(\mathbf{Q})(\geq 0),
$$

namely, $\sigma_{\mathbf{A}, \mathbf{B}}$ is a monotonic nondecreasing functional on $\mathcal{B}_{+}^{(n)}(H)$;

(iii) If $\mathbf{P}, \mathbf{Q} \in \mathcal{B}_{+}^{(n)}(H)$ and there exist the constants $M>m>0$ such that $M \mathbf{Q} \geq$ $\mathbf{P} \geq m \mathbf{Q}$ then

$$
M \sigma_{\mathbf{A}, \mathbf{B}}(\mathbf{Q}) \geq \sigma_{\mathbf{A}, \mathbf{B}}(\mathbf{P}) \geq m \sigma_{\mathbf{A}, \mathbf{B}}(\mathbf{Q})(\geq 0) .
$$

If $\mathbf{P}=\left(p_{1} 1_{H}, \ldots, p_{n} 1_{H}\right)$ with $p_{k} \geq 0, k \in\{1, \ldots, n\}$ then the functional of nonnegative weights $\mathbf{p}=\left(p_{1}, \ldots, p_{n}\right)$ defined by

$$
\sigma_{\mathbf{A}, \mathbf{B}}(\mathbf{p}):=\left[\operatorname{tr}\left(\sum_{k=1}^{n} p_{k}\left|A_{k}\right|^{2}\right)\right]^{\frac{1}{2}}\left[\operatorname{tr}\left(\sum_{k=1}^{n} p_{k}\left|B_{k}\right|^{2}\right)\right]^{\frac{1}{2}}-\left|\operatorname{tr}\left(\sum_{k=1}^{n} p_{k} A_{k}^{*} B_{k}\right)\right| .
$$

has the same properties as in (4.3)-(4.5).

Moreover, we have the simple bounds:

$$
\begin{aligned}
& \max _{k \in\{1, \ldots, n\}}\left\{p_{k}\right\}\left\{\left[\operatorname{tr}\left(\sum_{k=1}^{n}\left|A_{k}\right|^{2}\right)\right]^{\frac{1}{2}}\left[\operatorname{tr}\left(\sum_{k=1}^{n}\left|B_{k}\right|^{2}\right)\right]^{\frac{1}{2}}-\left|\operatorname{tr}\left(\sum_{k=1}^{n} A_{k}^{*} B_{k}\right)\right|\right\} \\
\geq & {\left[\operatorname{tr}\left(\sum_{k=1}^{n} p_{k}\left|A_{k}\right|^{2}\right)\right]^{\frac{1}{2}}\left[\operatorname{tr}\left(\sum_{k=1}^{n} p_{k}\left|B_{k}\right|^{2}\right)\right]^{\frac{1}{2}}-\left|\operatorname{tr}\left(\sum_{k=1}^{n} p_{k} A_{k}^{*} B_{k}\right)\right| } \\
\geq & \min _{k \in\{1, \ldots, n\}}\left\{p_{k}\right\}\left\{\left[\operatorname{tr}\left(\sum_{k=1}^{n}\left|A_{k}\right|^{2}\right)\right]^{\frac{1}{2}}\left[\operatorname{tr}\left(\sum_{k=1}^{n}\left|B_{k}\right|^{2}\right)\right]^{\frac{1}{2}}-\left|\operatorname{tr}\left(\sum_{k=1}^{n} A_{k}^{*} B_{k}\right)\right|\right\} .
\end{aligned}
$$




\section{Inequalities for Power Series of Operators}

Denote by:

$$
D(0, R)= \begin{cases}\{z \in \mathbb{C}:|z|<R\}, & \text { if } R<\infty \\ \mathbb{C}, & \text { if } R=\infty\end{cases}
$$

and consider the functions

$$
\lambda \mapsto f(\lambda): D(0, R) \rightarrow \mathbb{C}, f(\lambda):=\sum_{n=0}^{\infty} \alpha_{n} \lambda^{n}
$$

and

$$
\lambda \mapsto f_{a}(\lambda): D(0, R) \rightarrow \mathbb{C}, f_{a}(\lambda):=\sum_{n=0}^{\infty}\left|\alpha_{n}\right| \lambda^{n} .
$$

As some natural examples that are useful for applications, we can point out that, if

$$
\begin{aligned}
& f(\lambda)=\sum_{n=1}^{\infty} \frac{(-1)^{n}}{n} \lambda^{n}=\ln \frac{1}{1+\lambda}, \quad \lambda \in D(0,1) ; \\
& g(\lambda)=\sum_{n=0}^{\infty} \frac{(-1)^{n}}{(2 n) !} \lambda^{2 n}=\cos \lambda, \quad \lambda \in \mathbb{C} ; \\
& h(\lambda)=\sum_{n=0}^{\infty} \frac{(-1)^{n}}{(2 n+1) !} \lambda^{2 n+1}=\sin \lambda, \quad \lambda \in \mathbb{C} ; \\
& l(\lambda)=\sum_{n=0}^{\infty}(-1)^{n} \lambda^{n}=\frac{1}{1+\lambda}, \quad \lambda \in D(0,1) ;
\end{aligned}
$$

then the corresponding functions constructed by the use of the absolute values of the coefficients are

$$
\begin{aligned}
& f_{a}(\lambda)=\sum_{n=1}^{\infty} \frac{1}{n} \lambda^{n}=\ln \frac{1}{1-\lambda}, \quad \lambda \in D(0,1) ; \\
& g_{a}(\lambda)=\sum_{n=0}^{\infty} \frac{1}{(2 n) !} \lambda^{2 n}=\cosh \lambda, \quad \lambda \in \mathbb{C} ; \\
& h_{a}(\lambda)=\sum_{n=0}^{\infty} \frac{1}{(2 n+1) !} \lambda^{2 n+1}=\sinh \lambda, \quad \lambda \in \mathbb{C} ; \\
& l_{a}(\lambda)=\sum_{n=0}^{\infty} \lambda^{n}=\frac{1}{1-\lambda}, \quad \lambda \in D(0,1) .
\end{aligned}
$$

Other important examples of functions as power series representations with nonnegative coefficients are:

$$
\exp (\lambda)=\sum_{n=0}^{\infty} \frac{1}{n !} \lambda^{n}, \quad \lambda \in \mathbb{C}
$$




$$
\begin{aligned}
\frac{1}{2} \ln \left(\frac{1+\lambda}{1-\lambda}\right) & =\sum_{n=1}^{\infty} \frac{1}{2 n-1} \lambda^{2 n-1}, \quad \lambda \in D(0,1) \\
\sin ^{-1}(\lambda) & =\sum_{n=0}^{\infty} \frac{\Gamma\left(n+\frac{1}{2}\right)}{\sqrt{\pi}(2 n+1) n !} \lambda^{2 n+1}, \quad \lambda \in D(0,1) ; \\
\tanh ^{-1}(\lambda) & =\sum_{n=1}^{\infty} \frac{1}{2 n-1} \lambda^{2 n-1}, \quad \lambda \in D(0,1) ; \\
{ }_{2} F_{1}(\alpha, \beta, \gamma, \lambda) & =\sum_{n=0}^{\infty} \frac{\Gamma(n+\alpha) \Gamma(n+\beta) \Gamma(\gamma)}{n ! \Gamma(\alpha) \Gamma(\beta) \Gamma(n+\gamma)} \lambda^{n}, \quad \alpha, \beta, \gamma>0, \quad \lambda \in D(0,1) ;
\end{aligned}
$$

where $\Gamma$ is Gamma function.

Proposition 5.1. Let $f(\lambda):=\sum_{n=0}^{\infty} \alpha_{n} \lambda^{n}$ be a power series with complex coeffcients and convergent on the open disk $D(0, R), R>0$. If $(H,\langle\cdot, \cdot\rangle)$ is a separable infinite-dimensional Hilbert space and $A, B \in \mathcal{B}_{1}(H)$ are positive operators with $\operatorname{tr}(A)$, $\operatorname{tr}(B)<R^{\frac{1}{2}}$, then

$$
|\operatorname{tr}(f(A B))|^{2} \leq f_{a}^{2}(\operatorname{tr} A \operatorname{tr} B) \leq f_{a}\left((\operatorname{tr} A)^{2}\right) f_{a}\left((\operatorname{tr} B)^{2}\right) .
$$

Proof. By the inequality (1.4) for the positive operators $A, B \in \mathcal{B}_{1}(H)$ we have

$$
\begin{aligned}
\left|\operatorname{tr}\left[\sum_{k=0}^{n} \alpha_{k}(A B)^{k}\right]\right| & =\left|\sum_{k=0}^{n} \alpha_{k} \operatorname{tr}\left[(A B)^{k}\right]\right| \\
& \leq \sum_{k=0}^{n}\left|\alpha_{k}\right|\left|\operatorname{tr}\left[(A B)^{k}\right]\right|=\sum_{k=0}^{n}\left|\alpha_{k}\right| \operatorname{tr}\left[(A B)^{k}\right] \\
& \leq \sum_{k=0}^{n}\left|\alpha_{k}\right|(\operatorname{tr} A)^{k}(\operatorname{tr} B)^{k}=\sum_{k=0}^{n}\left|\alpha_{k}\right|(\operatorname{tr} A \operatorname{tr} B)^{k} .
\end{aligned}
$$

Utilizing the weighted Cauchy-Bunyakovsky-Schwarz inequality for sums we have

$$
\sum_{k=0}^{n}\left|\alpha_{k}\right|(\operatorname{tr} A)^{k}(\operatorname{tr} B)^{k} \leq\left(\sum_{k=0}^{n}\left|\alpha_{k}\right|(\operatorname{tr} A)^{2 k}\right)^{\frac{1}{2}}\left(\sum_{k=0}^{n}\left|\alpha_{k}\right|(\operatorname{tr} B)^{2 k}\right)^{\frac{1}{2}} .
$$

Then by (5.4) and (5.5) we have

$$
\begin{aligned}
\left|\operatorname{tr}\left[\sum_{k=0}^{n} \alpha_{k}(A B)^{k}\right]\right|^{2} & \leq\left[\sum_{k=0}^{n}\left|\alpha_{k}\right|(\operatorname{tr} A \operatorname{tr} B)^{k}\right]^{2} \\
& \leq \sum_{k=0}^{n}\left|\alpha_{k}\right|\left[(\operatorname{tr} A)^{2}\right]^{k} \sum_{k=0}^{n}\left|\alpha_{k}\right|\left[(\operatorname{tr} B)^{2}\right]^{k}
\end{aligned}
$$

for $n \geq 1$. 
Since $0 \leq \operatorname{tr}(A), \operatorname{tr}(B)<R^{\frac{1}{2}}$, the numerical series

$$
\sum_{k=0}^{\infty}\left|\alpha_{k}\right|(\operatorname{tr} A \operatorname{tr} B)^{k}, \quad \sum_{k=0}^{\infty}\left|\alpha_{k}\right|\left[(\operatorname{tr} A)^{2}\right]^{k} \quad \text { and } \sum_{k=0}^{\infty}\left|\alpha_{k}\right|\left[(\operatorname{tr} B)^{2}\right]^{k}
$$

are convergent.

Also, since $0 \leq \operatorname{tr}(A B) \leq \operatorname{tr}(A) \operatorname{tr}(B)<R$, the operator series $\sum_{k=0}^{\infty} \alpha_{k}(A B)^{k}$ is convergent in $\mathcal{B}_{1}(H)$.

Letting $n \rightarrow \infty$ in (5.6) and utilizing the continuity property of $\operatorname{tr}(\cdot)$ on $\mathcal{B}_{1}(H)$ we get the desired result (5.3).

Example 5.1.

a) If we take in (5.3) $f(\lambda)=(1 \pm \lambda)^{-1},|\lambda|<1$ then we get the inequality

$$
\left|\operatorname{tr}\left(\left(1_{H} \pm A B\right)^{-1}\right)\right|^{2} \leq\left(1-(\operatorname{tr} A)^{2}\right)^{-1}\left(1-(\operatorname{tr} B)^{2}\right)^{-1}
$$

for any $A, B \in \mathcal{B}_{1}(H)$ positive operators with $\operatorname{tr}(A), \operatorname{tr}(B)<1$.

b) If we take in (5.3) $f(\lambda)=\ln (1 \pm \lambda)^{-1},|\lambda|<1$, then we get the inequality

$$
\left|\operatorname{tr}\left(\ln \left(1_{H} \pm A B\right)^{-1}\right)\right|^{2} \leq \ln \left(1-(\operatorname{tr} A)^{2}\right)^{-1} \ln \left(1-(\operatorname{tr} B)^{2}\right)^{-1},
$$

for any $A, B \in \mathcal{B}_{1}(H)$ positive operators with $\operatorname{tr}(A), \operatorname{tr}(B)<1$.

We have the following result as well.

Theorem 5.1. Let $f(\lambda):=\sum_{n=0}^{\infty} \alpha_{n} \lambda^{n}$ be a power series with complex coefficients and convergent on the open disk $D(0, R), R>0$. If $A, B \in \mathcal{B}_{2}(H)$ are normal operators with $A^{*} B=B A^{*}$ and $\operatorname{tr}\left(|A|^{2}\right), \operatorname{tr}\left(|B|^{2}\right)<R$, then we have the inequality

$$
\left|\operatorname{tr}\left(f\left(A^{*} B\right)\right)\right|^{2} \leq \operatorname{tr}\left(f_{a}\left(|A|^{2}\right)\right) \operatorname{tr}\left(f_{a}\left(|B|^{2}\right)\right) .
$$

Proof. From the inequality (4.1) we have

$$
\left|\operatorname{tr}\left(\sum_{k=0}^{n} \alpha_{k}\left(A^{*}\right)^{k} B^{k}\right)\right|^{2} \leq \operatorname{tr}\left(\sum_{k=0}^{n}\left|\alpha_{k}\right|\left|A^{k}\right|^{2}\right) \operatorname{tr}\left(\sum_{k=0}^{n}\left|\alpha_{k}\right|\left|B^{k}\right|^{2}\right) .
$$

Since $A, B$ are normal operators, then we have $\left|A^{k}\right|^{2}=|A|^{2 k}$ and $\left|B^{k}\right|^{2}=|B|^{2 k}$ for any $k \geq 0$. Also, since $A^{*} B=B A^{*}$, then we also have $\left(A^{*}\right)^{k} B^{k}=\left(A^{*} B\right)^{k}$ for any $k \geq 0$.

Due to the fact that $A, B \in \mathcal{B}_{2}(H)$ and $\operatorname{tr}\left(|A|^{2}\right), \operatorname{tr}\left(|B|^{2}\right)<R$, it follows that $\operatorname{tr}\left(A^{*} B\right) \leq R$ and the operator series

$$
\sum_{k=0}^{\infty} \alpha_{k}\left(A^{*} B\right)^{k}, \quad \sum_{k=0}^{\infty}\left|\alpha_{k}\right||A|^{2 k} \text { and } \sum_{k=0}^{\infty}\left|\alpha_{k}\right||B|^{2 k}
$$

are convergent in the Banach space $\mathcal{B}_{1}(H)$.

Taking the limit over $n \rightarrow \infty$ in (5.9) and using the continuity of the $\operatorname{tr}(\cdot)$ on $\mathcal{B}_{1}(H)$ we deduce the desired result (5.8). 
Example 5.2.

a) If we take in $(5.8) f(\lambda)=(1 \pm \lambda)^{-1},|\lambda|<1$ then we get the inequality

$$
\left|\operatorname{tr}\left(\left(1_{H} \pm A^{*} B\right)^{-1}\right)\right|^{2} \leq \operatorname{tr}\left(\left(1-|A|^{2}\right)^{-1}\right) \operatorname{tr}\left(\left(1-|B|^{2}\right)^{-1}\right)
$$

for any $A, B \in \mathcal{B}_{2}(H)$ normal operators with $A^{*} B=B A^{*}$ and $\operatorname{tr}\left(|A|^{2}\right), \operatorname{tr}\left(|B|^{2}\right)<1$. b) If we take in (5.8) $f(\lambda)=\exp (\lambda), \lambda \in \mathbb{C}$ then we get the inequality

$$
\left|\operatorname{tr}\left(\exp \left(A^{*} B\right)\right)\right|^{2} \leq \operatorname{tr}\left(\exp \left(|A|^{2}\right)\right) \operatorname{tr}\left(\exp \left(|B|^{2}\right)\right)
$$

for any $A, B \in \mathcal{B}_{2}(H)$ normal operators with $A^{*} B=B A^{*}$.

Theorem 5.2. Let $f(z):=\sum_{j=0}^{\infty} p_{j} z^{j}$ and $g(z):=\sum_{j=0}^{\infty} q_{j} z^{j}$ be two power series with nonnegative coefficients and convergent on the open disk $D(0, R), R>0$. If $T$ and $V$ are two normal and commuting operators from $\mathcal{B}_{2}(H)$ with $\operatorname{tr}\left(|T|^{2}\right), \operatorname{tr}\left(|V|^{2}\right)<R$, then

$$
\begin{aligned}
& {\left[\operatorname{tr}\left(f\left(|T|^{2}\right)+g\left(|T|^{2}\right)\right)\right]^{\frac{1}{2}}\left[\operatorname{tr}\left(f\left(|V|^{2}\right)+g\left(|V|^{2}\right)\right)\right]^{\frac{1}{2}} } \\
& -\left|\operatorname{tr}\left(f\left(T^{*} V\right)+g\left(T^{*} V\right)\right)\right| \\
\geq & {\left[\operatorname{tr}\left(f\left(|T|^{2}\right)\right)\right]^{\frac{1}{2}}\left[\operatorname{tr}\left(f\left(|V|^{2}\right)\right)\right]^{\frac{1}{2}}-\left|\operatorname{tr}\left(f\left(T^{*} V\right)\right)\right| } \\
& +\left[\operatorname{tr}\left(g\left(|T|^{2}\right)\right)\right]^{\frac{1}{2}}\left[\operatorname{tr}\left(g\left(|V|^{2}\right)\right)\right]^{\frac{1}{2}}-\left|\operatorname{tr}\left(g\left(T^{*} V\right)\right)\right|(\geq 0) .
\end{aligned}
$$

Moreover, if $p_{j} \geq q_{j}$ for any $j \in \mathbb{N}$, then, with the above assumptions on $T$ and $V$, we have

$$
\begin{aligned}
& {\left[\operatorname{tr}\left(f\left(|T|^{2}\right)\right)\right]^{\frac{1}{2}}\left[\operatorname{tr}\left(f\left(|V|^{2}\right)\right)\right]^{\frac{1}{2}}-\left|\operatorname{tr}\left(f\left(T^{*} V\right)\right)\right| } \\
\geq & {\left[\operatorname{tr}\left(g\left(|T|^{2}\right)\right)\right]^{\frac{1}{2}}\left[\operatorname{tr}\left(g\left(|V|^{2}\right)\right)\right]^{\frac{1}{2}}-\left|\operatorname{tr}\left(g\left(T^{*} V\right)\right)\right|(\geq 0) . }
\end{aligned}
$$

Proof. Utilizing the superadditivity property of the functional $\sigma_{\mathbf{A}, \mathbf{B}}(\cdot)$ above as a function of weights $\mathbf{p}$ and the fact that $T$ and $V$ are two normal and commuting operators we can state that

$$
\begin{aligned}
& {\left[\operatorname{tr}\left(\sum_{k=0}^{n}\left(p_{k}+q_{k}\right)|T|^{2 k}\right)\right]^{\frac{1}{2}}\left[\operatorname{tr}\left(\sum_{k=0}^{n}\left(p_{k}+q_{k}\right)|V|^{2 k}\right)\right]^{\frac{1}{2}} } \\
& -\left|\operatorname{tr}\left(\sum_{k=0}^{n}\left(p_{k}+q_{k}\right)\left(T^{*} V\right)^{k}\right)\right| \\
\geq & {\left[\operatorname{tr}\left(\sum_{k=0}^{n} p_{k}|T|^{2 k}\right)\right]^{\frac{1}{2}}\left[\operatorname{tr}\left(\sum_{k=0}^{n} p_{k}|V|^{2 k}\right)\right]^{\frac{1}{2}}-\left|\operatorname{tr}\left(\sum_{k=0}^{n} p_{k}\left(T^{*} V\right)^{k}\right)\right| } \\
& +\left[\operatorname{tr}\left(\sum_{k=0}^{n} q_{k}|T|^{2 k}\right)\right]^{\frac{1}{2}}\left[\operatorname{tr}\left(\sum_{k=0}^{n} q_{k}|V|^{2 k}\right)\right]^{\frac{1}{2}}-\left|\operatorname{tr}\left(\sum_{k=0}^{n} q_{k}\left(T^{*} V\right)^{k}\right)\right|
\end{aligned}
$$

for any $n \geq 1$. 
Since all the series whose partial sums are involved in (5.13) are convergent in $\mathcal{B}_{1}(H)$, by letting $n \rightarrow \infty$ in (5.13) we get (5.11).

The inequality (5.12) follows by the monotonicity property of $\sigma_{\mathbf{A}, \mathbf{B}}(\cdot)$ and the details are omitted.

Example 5.3. Now, observe that if we take

$$
f(\lambda)=\sinh \lambda=\sum_{n=0}^{\infty} \frac{1}{(2 n+1) !} \lambda^{2 n+1}
$$

and

$$
g(\lambda)=\cosh \lambda=\sum_{n=0}^{\infty} \frac{1}{(2 n) !} \lambda^{2 n}
$$

then

$$
f(\lambda)+g(\lambda)=\exp \lambda=\sum_{n=0}^{\infty} \frac{1}{n !} \lambda^{n}
$$

for any $\lambda \in \mathbb{C}$.

If $T$ and $V$ are two normal and commuting operators from $\mathcal{B}_{2}(H)$, then by $(2.11)$ we have

$$
\begin{aligned}
& {\left[\operatorname{tr}\left(\exp \left(|T|^{2}\right)\right)\right]^{\frac{1}{2}}\left[\operatorname{tr}\left(\exp \left(|V|^{2}\right)\right)\right]^{\frac{1}{2}}-\left|\operatorname{tr}\left(\exp \left(T^{*} V\right)\right)\right| } \\
\geq & {\left[\operatorname{tr}\left(\sinh \left(|T|^{2}\right)\right)\right]^{\frac{1}{2}}\left[\operatorname{tr}\left(\sinh \left(|V|^{2}\right)\right)\right]^{\frac{1}{2}}-\left|\operatorname{tr}\left(\sinh \left(T^{*} V\right)\right)\right| } \\
& +\left[\operatorname{tr}\left(\cosh \left(|T|^{2}\right)\right)\right]^{\frac{1}{2}}\left[\operatorname{tr}\left(\cosh \left(|V|^{2}\right)\right)\right]^{\frac{1}{2}}-\left|\operatorname{tr}\left(\cosh \left(T^{*} V\right)\right)\right|(\geq 0) .
\end{aligned}
$$

Now, consider the series $\frac{1}{1-\lambda}=\sum_{n=0}^{\infty} \lambda^{n}, \lambda \in D(0,1)$ and $\ln \frac{1}{1-\lambda}=\sum_{n=1}^{\infty} \frac{1}{n} \lambda^{n}$, $\lambda \in D(0,1)$ and define $p_{n}=1, n \geq 0, q_{0}=0, q_{n}=\frac{1}{n}, n \geq 1$, then we observe that for any $n \geq 0$ we have $p_{n} \geq q_{n}$.

If $T$ and $V$ are two normal and commuting operators from $\mathcal{B}_{2}(H)$ with $\operatorname{tr}\left(|T|^{2}\right)$, $\operatorname{tr}\left(|V|^{2}\right)<1$, then by $(2.12)$ we have

$$
\begin{aligned}
& {\left[\operatorname{tr}\left(\left(1_{H}-|T|^{2}\right)^{-1}\right)\right]^{\frac{1}{2}}\left[\operatorname{tr}\left(\left(1_{H}-|V|^{2}\right)^{-1}\right)\right]^{\frac{1}{2}}-\left|\operatorname{tr}\left(\left(1_{H}-T^{*} V\right)^{-1}\right)\right| } \\
\geq & {\left[\operatorname{tr}\left(\ln \left(1_{H}-|T|^{2}\right)^{-1}\right)\right]^{\frac{1}{2}}\left[\operatorname{tr}\left(\ln \left(1_{H}-|V|^{2}\right)^{-1}\right)\right]^{\frac{1}{2}}-\left|\operatorname{tr}\left(\ln \left(1_{H}-T^{*} V\right)^{-1}\right)\right|(\geq 0) . }
\end{aligned}
$$

\section{INEQUALities FOR MATRICES}

We have the following result for matrices.

Proposition 6.1. Let $f(\lambda):=\sum_{n=0}^{\infty} \alpha_{n} \lambda^{n}$ be a power series with complex coefficients and convergent on the open disk $D(0, R), R>0$. If $A$ and $B$ are positive semidefinite matrices in $M_{n}(\mathbb{C})$ with $\operatorname{tr}\left(A^{2}\right), \operatorname{tr}\left(B^{2}\right)<R$, then we have the inequality

$$
|\operatorname{tr}[f(A B)]|^{2} \leq \operatorname{tr}\left[f_{a}\left(A^{2}\right)\right] \operatorname{tr}\left[f_{a}\left(B^{2}\right)\right] .
$$


If $\operatorname{tr}(A), \operatorname{tr}(B)<\sqrt{R}$, then also

$$
|\operatorname{tr}[f(A B)]| \leq \min \left\{\operatorname{tr}\left(f_{a}(\|A\| B)\right), \operatorname{tr}\left(f_{a}(\|B\| A)\right)\right\} .
$$

Proof. We observe that (1.5) holds for $m=0$ with equality.

By utilizing the generalized triangle inequality for the modulus and the inequality (1.5) we have

$$
\begin{aligned}
\left|\operatorname{tr}\left[\sum_{n=0}^{m} \alpha_{n}(A B)^{n}\right]\right| & =\left|\sum_{n=0}^{m} \alpha_{n} \operatorname{tr}\left[(A B)^{n}\right]\right| \leq \sum_{n=0}^{m}\left|\alpha_{n}\right|\left|\operatorname{tr}\left[(A B)^{n}\right]\right| \\
& =\sum_{n=0}^{m}\left|\alpha_{n}\right| \operatorname{tr}\left[(A B)^{n}\right] \leq \sum_{n=0}^{m}\left|\alpha_{n}\right|\left[\operatorname{tr}\left(A^{2 n}\right)\right]^{\frac{1}{2}}\left[\operatorname{tr}\left(B^{2 n}\right)\right]^{\frac{1}{2}}
\end{aligned}
$$

for any $m \geq 1$.

Applying the weighted Cauchy-Bunyakowsky-Schwarz discrete inequality we also have

$$
\begin{aligned}
& \sum_{n=0}^{m}\left|\alpha_{n}\right|\left[\operatorname{tr}\left(A^{2 n}\right)\right]^{\frac{1}{2}}\left[\operatorname{tr}\left(B^{2 n}\right)\right]^{\frac{1}{2}} \\
\leq & \left(\sum_{n=0}^{m}\left|\alpha_{n}\right|\left(\left[\operatorname{tr}\left(A^{2 n}\right)\right]^{\frac{1}{2}}\right)^{2}\right)^{\frac{1}{2}}\left(\sum_{n=0}^{m}\left|\alpha_{n}\right|\left(\left[\operatorname{tr}\left(B^{2 n}\right)\right]^{\frac{1}{2}}\right)^{2}\right)^{\frac{1}{2}} \\
= & \left(\sum_{n=0}^{m}\left|\alpha_{n}\right|\left[\operatorname{tr}\left(A^{2 n}\right)\right]\right)^{\frac{1}{2}}\left(\sum_{n=0}^{m}\left|\alpha_{n}\right|\left[\operatorname{tr}\left(B^{2 n}\right)\right]\right)^{\frac{1}{2}} \\
= & {\left[\operatorname{tr}\left(\sum_{n=0}^{m}\left|\alpha_{n}\right| A^{2 n}\right)\right]^{\frac{1}{2}}\left[\operatorname{tr}\left(\sum_{n=0}^{m}\left|\alpha_{n}\right| B^{2 n}\right)\right]^{\frac{1}{2}} }
\end{aligned}
$$

for any $m \geq 1$.

Therefore, by (6.3) and (6.4) we get

$$
\left|\operatorname{tr}\left[\sum_{n=0}^{m} \alpha_{n}(A B)^{n}\right]\right|^{2} \leq \operatorname{tr}\left(\sum_{n=0}^{m}\left|\alpha_{n}\right| A^{2 n}\right) \operatorname{tr}\left(\sum_{n=0}^{m}\left|\alpha_{n}\right| B^{2 n}\right)
$$

for any $m \geq 1$.

Since $\operatorname{tr}\left(A^{2}\right), \operatorname{tr}\left(B^{2}\right)<R$, then $\operatorname{tr}(A B) \leq \sqrt{\operatorname{tr}\left(A^{2}\right) \operatorname{tr}\left(B^{2}\right)}<R$ and the series

$$
\sum_{n=0}^{\infty} \alpha_{n}(A B)^{n}, \quad \sum_{n=0}^{\infty}\left|\alpha_{n}\right| A^{2 n} \text { and } \sum_{n=0}^{\infty}\left|\alpha_{n}\right| B^{2 n}
$$

are convergent in $M_{n}(\mathbb{C})$.

Taking the limit over $m \rightarrow \infty$ in (6.5) and utilizing the continuity property of $\operatorname{tr}(\cdot)$ on $M_{n}(\mathbb{C})$ we get $(6.1)$.

The inequality (6.2) follows from (1.6) in a similar way and the details are omitted. 
Example 6.1.

a) If we take $f(\lambda)=(1 \pm \lambda)^{-1},|\lambda|<1$ then we get the inequality

$$
\left|\operatorname{tr}\left[\left(I_{n} \pm A B\right)^{-1}\right]\right|^{2} \leq \operatorname{tr}\left[\left(I_{n}-A^{2}\right)^{-1}\right] \operatorname{tr}\left[\left(I_{n}-B^{2}\right)^{-1}\right]
$$

for any $A$ and $B$ positive semidefinite matrices in $M_{n}(\mathbb{C})$ with $\operatorname{tr}\left(A^{2}\right), \operatorname{tr}\left(B^{2}\right)<1$. Here $I_{n}$ is the identity matrix in $M_{n}(\mathbb{C})$.

We also have the inequality

$$
\left|\operatorname{tr}\left[\left(I_{n} \pm A B\right)^{-1}\right]\right| \leq \min \left\{\operatorname{tr}\left(\left(I_{n}-\|A\| B\right)^{-1}\right), \operatorname{tr}\left(\left(I_{n}-\|B\| A\right)^{-1}\right)\right\}
$$

for any $A$ and $B$ positive semidefinite matrices in $M_{n}(\mathbb{C})$ with $\operatorname{tr}(A), \operatorname{tr}(B)<1$.

b) If we take $f(\lambda)=\exp \lambda$, then we have the inequalities

$$
(\operatorname{tr}[\exp (A B)])^{2} \leq \operatorname{tr}\left[\exp \left(A^{2}\right)\right] \operatorname{tr}\left[\exp \left(B^{2}\right)\right]
$$

and

$$
\operatorname{tr}[\exp (A B)] \leq \min \{\operatorname{tr}(\exp (\|A\| B)), \operatorname{tr}(\exp (\|B\| A))\}
$$

for any $A$ and $B$ positive semidefinite matrices in $M_{n}(\mathbb{C})$.

Proposition 6.2. Let $f(\lambda):=\sum_{n=0}^{\infty} \alpha_{n} \lambda^{n}$ be a power series with complex coefficients and convergent on the open disk $D(0, R), R>0$. If $A$ and $B$ are matrices in $M_{n}(\mathbb{C})$ with $\operatorname{tr}\left(|A|^{p}\right), \operatorname{tr}\left(|B|^{q}\right)<R$, where $p, q>1$ with $1 / p+1 / q=1$, then

$$
\left|\operatorname{tr}\left(f\left(\left|A B^{*}\right|\right)\right)\right| \leq \operatorname{tr}\left[f_{a}\left(\frac{|A|^{p}}{p}+\frac{|B|^{q}}{q}\right)\right] \leq \operatorname{tr}\left[\frac{1}{p} f_{a}\left(|A|^{p}\right)+\frac{1}{q} f_{a}\left(|B|^{q}\right)\right] .
$$

Proof. The inequality (1.7) holds with equality for $r=0$.

By utilizing the generalized triangle inequality for the modulus and the inequality (1.7) we have

$$
\begin{aligned}
\left|\operatorname{tr}\left(\sum_{n=0}^{m} \alpha_{n}\left|A B^{*}\right|^{n}\right)\right| & =\left|\sum_{n=0}^{m} \alpha_{n} \operatorname{tr}\left(\left|A B^{*}\right|^{n}\right)\right| \\
& \leq \sum_{n=0}^{m}\left|\alpha_{n}\right|\left|\operatorname{tr}\left(\left|A B^{*}\right|^{n}\right)\right|=\sum_{n=0}^{m}\left|\alpha_{n}\right| \operatorname{tr}\left(\left|A B^{*}\right|^{n}\right) \\
& \leq \sum_{n=0}^{m}\left|\alpha_{n}\right| \operatorname{tr}\left[\left(\frac{|A|^{p}}{p}+\frac{|B|^{q}}{q}\right)^{n}\right] \\
& =\operatorname{tr}\left[\sum_{n=0}^{m}\left|\alpha_{n}\right|\left(\frac{|A|^{p}}{p}+\frac{|B|^{q}}{q}\right)^{n}\right]
\end{aligned}
$$

for any $m \geq 1$ and $p, q>1$ with $1 / p+1 / q=1$.

It is known that if $f:[0, \infty) \rightarrow \mathbb{R}$ is a convex function, then $\operatorname{tr} f(\cdot)$ is convex on the cone $M_{n}^{+}(\mathbb{C})$ of positive semidefinite matrices in $M_{n}(\mathbb{C})$. Therefore, for $n \geq 1$ we have

$$
\operatorname{tr}\left[\left(\frac{|A|^{p}}{p}+\frac{|B|^{q}}{q}\right)^{n}\right] \leq \frac{1}{p} \operatorname{tr}\left(|A|^{p n}\right)+\frac{1}{q} \operatorname{tr}\left(|B|^{q n}\right)
$$


where $p, q>1$ with $1 / p+1 / q=1$.

The inequality reduces to equality if $n=0$.

Then we have

$$
\begin{aligned}
\sum_{n=0}^{m}\left|\alpha_{n}\right| \operatorname{tr}\left[\left(\frac{|A|^{p}}{p}+\frac{|B|^{q}}{q}\right)^{n}\right] & \leq \sum_{n=0}^{m}\left|\alpha_{n}\right|\left[\frac{1}{p} \operatorname{tr}\left(|A|^{p n}\right)+\frac{1}{q} \operatorname{tr}\left(|B|^{q n}\right)\right] \\
& =\operatorname{tr}\left[\frac{1}{p} \sum_{n=0}^{m}\left|\alpha_{n}\right||A|^{p n}+\frac{1}{q} \sum_{n=0}^{m}\left|\alpha_{n}\right||B|^{q n}\right]
\end{aligned}
$$

for any $m \geq 1$ and $p, q>1$ with $1 / p+1 / q=1$.

From (6.7) and (6.8) we get

$$
\begin{aligned}
\left|\operatorname{tr}\left(\sum_{n=0}^{m} \alpha_{n}\left|A B^{*}\right|^{r}\right)\right| & \leq \operatorname{tr}\left[\sum_{n=0}^{m}\left|\alpha_{n}\right|\left(\frac{|A|^{p}}{p}+\frac{|B|^{q}}{q}\right)^{n}\right] \\
& \leq \operatorname{tr}\left[\frac{1}{p} \sum_{n=0}^{m}\left|\alpha_{n}\right||A|^{p n}+\frac{1}{q} \sum_{n=0}^{m}\left|\alpha_{n}\right||B|^{q n}\right]
\end{aligned}
$$

for any $m \geq 1$ and $p, q>1$ with $1 / p+1 / q=1$.

Since $\operatorname{tr}\left(|A|^{p}\right), \operatorname{tr}\left(|B|^{q}\right)<R$, then all the series whose partial sums are involved in (6.9) are convergent, then by letting $m \rightarrow \infty$ in (6.9) we deduce the desired inequality (6.6).

Example 6.2.

a) If we take $f(\lambda)=(1 \pm \lambda)^{-1},|\lambda|<1$ then we get the inequalities

$$
\begin{aligned}
\left|\operatorname{tr}\left(\left(I_{n} \pm\left|A B^{*}\right|\right)^{-1}\right)\right| & \leq \operatorname{tr}\left(\left[I_{n}-\left(\frac{|A|^{p}}{p}+\frac{|B|^{q}}{q}\right)\right]^{-1}\right) \\
& \leq \operatorname{tr}\left[\frac{1}{p}\left(I_{n}-|A|^{p}\right)^{-1}+\frac{1}{q}\left(I_{n}-|B|^{q}\right)^{-1}\right],
\end{aligned}
$$

where $A$ and $B$ are matrices in $M_{n}(\mathbb{C})$ with $\operatorname{tr}\left(|A|^{p}\right), \operatorname{tr}\left(|B|^{q}\right)<1$ and $p, q>1$ with $1 / p+1 / q=1$.

b) If we take $f(\lambda)=\exp \lambda$, then we have the inequalities

$$
\begin{aligned}
\operatorname{tr}\left(\exp \left(\left|A B^{*}\right|\right)\right) & \leq \operatorname{tr}\left[\exp \left(\frac{|A|^{p}}{p}+\frac{|B|^{q}}{q}\right)\right] \\
& \leq \operatorname{tr}\left[\frac{1}{p} \exp \left(|A|^{p}\right)+\frac{1}{q} \exp \left(|B|^{q}\right)\right]
\end{aligned}
$$

where $A$ and $B$ are matrices in $M_{n}(\mathbb{C})$ and $p, q>1$ with $1 / p+1 / q=1$.

Finally, we can state the following result.

Proposition 6.3. Let $f(\lambda):=\sum_{n=0}^{\infty} \alpha_{n} \lambda^{n}$ be a power series with complex coefficients and convergent on the open disk $D(0, R), R>0$. If $A$ and $B$ are commuting positive 
semidefinite matrices in $M_{n}(\mathbb{C})$ with $\operatorname{tr}\left(A^{p}\right), \operatorname{tr}\left(B^{q}\right)<R$, where $p, q>1$ with $1 / p+$ $1 / q=1$, then we have the inequality

$$
|\operatorname{tr}(f(A B))| \leq\left[\operatorname{tr}\left(f_{a}\left(A^{p}\right)\right)\right]^{\frac{1}{p}}\left[\operatorname{tr}\left(f_{a}\left(B^{q}\right)\right)\right]^{\frac{1}{q}} .
$$

Proof. Since $A$ and $B$ are commuting positive semidefinite matrices in $M_{n}(\mathbb{C})$, then by (1.8) we have for any natural number $n$ including $n=0$ that

$$
\operatorname{tr}\left((A B)^{n}\right)=\operatorname{tr}\left(A^{n} B^{n}\right) \leq\left[\operatorname{tr}\left(A^{n p}\right)\right]^{\frac{1}{p}}\left[\operatorname{tr}\left(B^{n q}\right)\right]^{\frac{1}{q}},
$$

where $p, q>1$ with $\frac{1}{p}+\frac{1}{q}=1$.

By (6.10) and the weighted Hölder discrete inequality we have

$$
\begin{aligned}
\left|\operatorname{tr}\left(\sum_{n=0}^{m} \alpha_{n}(A B)^{n}\right)\right| & =\left|\sum_{n=0}^{m} \alpha_{n} \operatorname{tr}\left(A^{n} B^{n}\right)\right| \leq \sum_{n=0}^{m}\left|\alpha_{n}\right|\left|\operatorname{tr}\left(A^{n} B^{n}\right)\right| \\
& \leq \sum_{n=0}^{m}\left|\alpha_{n}\right|\left[\operatorname{tr}\left(A^{n p}\right)\right]^{\frac{1}{p}}\left[\operatorname{tr}\left(B^{n q}\right)\right]^{\frac{1}{q}} \\
& \leq\left(\sum_{n=0}^{m}\left|\alpha_{n}\right|\left(\left[\operatorname{tr}\left(A^{n p}\right)\right]^{\frac{1}{p}}\right)^{p}\right)^{\frac{1}{p}}\left(\sum_{n=0}^{m}\left|\alpha_{n}\right|\left(\left[\operatorname{tr}\left(B^{n q}\right)\right]^{\frac{1}{q}}\right)^{q}\right)^{\frac{1}{q}} \\
& =\left(\sum_{n=0}^{m}\left|\alpha_{n}\right| \operatorname{tr}\left(A^{n p}\right)\right)^{\frac{1}{p}}\left(\sum_{n=0}^{m}\left|\alpha_{n}\right| \operatorname{tr}\left(B^{n q}\right)\right)^{\frac{1}{q}} \\
& =\left(\operatorname{tr}\left(\sum_{n=0}^{m}\left|\alpha_{n}\right| A^{n p}\right)\right)^{\frac{1}{p}}\left(\operatorname{tr}\left(\sum_{n=0}^{m}\left|\alpha_{n}\right| B^{n q}\right)\right)^{\frac{1}{q}},
\end{aligned}
$$

where $p, q>1$ with $1 / p+1 / q=1$.

The proof follows now in a similar way with the ones from above and the details are omitted.

\section{Example 6.3.}

a) If we take $f(\lambda)=(1 \pm \lambda)^{-1},|\lambda|<1$ then we get the inequality

$$
\left|\operatorname{tr}\left(\left(I_{n} \pm A B\right)^{-1}\right)\right| \leq\left[\operatorname{tr}\left(\left(I_{n}-A^{p}\right)^{-1}\right)\right]^{\frac{1}{p}}\left[\operatorname{tr}\left(\left(I_{n}-B^{q}\right)^{-1}\right)\right]^{\frac{1}{q}},
$$

for any $A$ and $B$ commuting positive semidefinite matrices in $M_{n}(\mathbb{C})$ with $\operatorname{tr}\left(A^{p}\right)$, $\operatorname{tr}\left(B^{q}\right)<1$, where $p, q>1$ with $1 / p+1 / q=1$.

b) If we take $f(\lambda)=\exp \lambda$, then we have the inequality

$$
\operatorname{tr}(\exp (A B)) \leq\left[\operatorname{tr}\left(\exp \left(A^{p}\right)\right)\right]^{\frac{1}{p}}\left[\operatorname{tr}\left(\exp \left(B^{q}\right)\right)\right]^{\frac{1}{q}},
$$

for any $A$ and $B$ commuting positive semidefinite matrices in $M_{n}(\mathbb{C})$ and $p, q>1$ with $1 / p+1 / q=1$. 


\section{REFERENCES}

[1] T. Ando, Matrix young inequalities, Oper. Theory Adv. Appl. 75 (1995), 33-38.

[2] R. Bellman, Some inequalities for positive definite matrices, in: E. F. Beckenbach (Ed.), Proceedings of the 2nd International Conference on General Inequalities, Birkhäuser, Basel, 1980, pp. 89-90.

[3] D. Chang, A matrix trace inequality for products of Hermitian matrices, J. Math. Anal. Appl. 237 (1999), 721-725.

[4] I. D. Coop, On matrix trace inequalities and related topics for products of hermitian matrix, J. Math. Anal. Appl. 188 (1994), 999-1001.

[5] N. G. de Bruijn, Problem 12, Wisk. Opgaven 21 (1960), 12-14.

[6] E.-V. Belmega, M. Jungers and S. Lasaulce, A generalization of a trace inequality for positive definite matrices, Aust. J. Math. Anal. Appl. 7 (2010), 1-5.

[7] S. Furuichi and M. Lin, Refinements of the trace inequality of Belmega, Lasaulce and Debbah, Aust. J. Math. Anal. Appl. 7 (2010), 1-4.

[8] H. D. Lee, On some matrix inequalities, Korean J. Math. 16 (2008), 565-571.

[9] L. Liu, A trace class operator inequality, J. Math. Anal. Appl. 328 (2007), 1484-1486.

[10] S. Manjegani, Hölder and young inequalities for the trace of operators, Positivity 11 (2007), 239-250.

[11] H. Neudecker, A matrix trace inequality, J. Math. Anal. Appl. 166 (1992), 302-303.

[12] K. Shebrawi and H. Albadawi, Operator norm inequalities of Minkowski type, J. Inequal. Pure Appl. Math. 9 (2008), 1-10.

[13] K. Shebrawi and H. Albadawi, Trace inequalities for matrices, Bull. Aust. Math. Soc. 87 (2013), 139-148.

[14] B. Simon, Trace Ideals and their Applications, Cambridge University Press, Cambridge, 1979.

[15] Z. Ulukök and R. Türkmen, On some matrix trace inequalities, J. Inequal. Appl. 2010 (2010), $1-8$.

[16] X. M. Yang, X. Q. Yang and K. L. Teo, A matrix trace inequality, J. Math. Anal. Appl. 263 (2001), 327-331.

[17] X. Yang, A matrix trace inequality, J. Math. Anal. Appl. 250 (2000), 372-374.

[18] Y. Yang, A matrix trace inequality, J. Math. Anal. Appl. 133 (1988), 573-574.

${ }^{1}$ Mathematics, School of Engineering \& Science

Victoria UniVERSITY

PO Box 14428, Melbourne City, MC 8001

Australia

E-mail address: sever.dragomir@vu.edu.au

${ }^{2}$ DST-NRF Centre of Excellence in the Mathematical and Statistical Sciences

School of Computational \& Applied Mathematics

UNIVERSITY OF THE WiTWATERSRAND

Private Bag 3, Johannesburg 2050

SOUTh AFrica 